\title{
Reflets
}

Revue ontaroise d'intervention sociale et communautaire

\section{Le système de coordination des services, un modèle à suivre}

\section{Carole Groulx}

Volume 4, numéro 2, automne 1998

Personnes vivant avec une incapacité

URI : https://id.erudit.org/iderudit/026235ar

DOI : https://doi.org/10.7202/026235ar

Aller au sommaire du numéro

Éditeur(s)

Reflets : Revue ontaroise d'intervention sociale et communautaire

ISSN

1203-4576 (imprimé)

1712-8498 (numérique)

Découvrir la revue

Citer cet article

Groulx, C. (1998). Le système de coordination des services, un modèle à suivre. Reflets, 4(2), 201-206. https://doi.org/10.7202/026235ar

Tous droits réservés (C) Reflets : Revue ontaroise d'intervention sociale et communautaire, 1998
Ce document est protégé par la loi sur le droit d'auteur. L'utilisation des services d’Érudit (y compris la reproduction) est assujettie à sa politique d'utilisation que vous pouvez consulter en ligne.

https://apropos.erudit.org/fr/usagers/politique-dutilisation/ 


\section{Le système de coordination des services, un modèle à suivre}

\section{Carole Groulx}

texte traduit par Jocelyne Lambert

Le système de coordination des services de Sudbury peut maintenant servir de modèle aux organismes de la province qui souhaitent offrir un point d'accès unique pour tous les services touchant les personnes ayant un handicap. En effet, le système que gère laYWCA de Sudbury est l'un des rares modèles d'accès direct aux services qui existent actuellement en Ontario ${ }^{1}$.

\section{Ce qu'est un système de coordination des services}

Un système de coordination des services permet aux personnes qui ont un handicap et à leur famille d'identifier, d'utiliser et de coordonner, en fonction de leurs besoins particuliers, les différentes ressources disponibles dans leur collectivité. Il fournit un accès direct à tous les services offerts aux personnes ayant un handicap dans les districts de Sudbury-Manitoulin. Comme il s'agit d'un modèle de prestation de services autonomes, les coordonnatrices de services peuvent intervenir selon les préférences et les besoins exprimés par le client et non selon les besoins et les contraintes fixés par la collectivité. 


\section{Éléments essentiels d'un système de coordination des services}

Un système de coordination des services comporte les éléments suivants :

- un service d'accueil servant à déterminer l'admissibilité;

- un processus servant à jumeler un client admissible et une coordonnatrice de services qui recueille auprès du client des renseignements sur ses besoins et ses préférences par rapport aux services;

- des négociations avec les organismes et la conclusion d'ententes relatives à la prestation des services nécessaires à un client;

- la coordination de mécanismes de soutien naturel et d'accès pour guider le client dans le labyrinthe de services tels que les Associations pour l'intégration communautaire, L'Arche, Christian Horizons, les services de relève, etc.;

- une base de données contenant des statistiques générales sur les besoins des habitants de la région;

- un service de gestion de cas offert aux clients tout au long des différentes étapes de leur trajectoire de vie afin que ceux-ci puissent se retrouver dans un système actuellement fragmenté de pourvoyeurs de services. En effet, la coordination des services ne cesse pas lorsque le client atteint un certain âge, ce qui permet l'établissement d'une planification à long terme;

- la gestion de crises pour les clients, lorsque cela s'avère nécessaire;

- l'établissement de liens avec les pourvoyeurs des services aux personnes ayant un handicap, comme le Centre d'accès aux soins communautaires, les services d'orthophonie et les services de relève. 


\section{Vision du système de coordination}

Le système de coordination des services cherche à offrir à tous ses clients une meilleure qualité de la vie par une planification axée sur la personne et une meilleure accessibilité aux services. Il a pour objectif de venir en aide aux personnes ayant un handicap et de leur permettre de se prendre en main. Il vise également à sensibiliser les membres de la collectivité et à les encourager à s'entraider.

\section{Rôle de la coordonnatrice de services}

La coordonnatrice de services aide les personnes ayant un handicap et leurs familles à déterminer et à obtenir les services dont elles ont besoin. Elle favorise aussi l'établissement de mécanismes de soutien naturel. De plus, elle intervient au nom des familles, elle suit leurs progrès et elle les aide à évaluer les services. L'une des responsabilités principales de la coordonnatrice de services consiste à guider les clients dans le processus menant à l'obtention des services et à les aider à remplir les formulaires nécessaires.

Le réseau regroupant les nombreux organismes offrant des services aux personnes ayant un handicap peut s'avérer complexe. On peut facilement s'y perdre. Il arrive souvent que la famille soit obligée de recueillir des renseignements, de remplir des formulaires, de fixer des rendez-vous et de visiter différents endroits avant d'obtenir le service dont un de ses membres a besoin. Tout cela peut prendre beaucoup de temps et être frustrant. La coordonnatrice de services fournit aux clients un point d'accès unique aux services.

De plus, la coordonnatrice de services joue un rôle important dans la compilation de statistiques qu'elle recueille en communiquant avec les clients et les familles. Elle obtient aussi fréquemment des informations sur ces clients auprès des 
pourvoyeurs de services. Elle participe également aux réunions de planification axée sur la personne et aux conférences de cas. Pour la collectivité, ces statistiques facilitent la planification à long terme en matière de services et de soutien aux personnes ayant un handicap.

\section{Rôles du client et de la famille dans la coordination des services}

On adapte les services aux besoins du client et de sa famille plutôt que d'adapter les besoins du client aux services qui existent déjà. La coordonnatrice de services utilise les ressources formelles et informelles (famille, amis, collègues de travail, voisins, etc.) du milieu.

C'est toujours la personne ou la famille que demande de l'aide en matière de coordination des services et qui prend la décision finale en tout temps. Elles peuvent, entre autres:

- décider quand elles veulent participer au système et quand elles veulent s'en retirer;

- décider si elles veulent participer à un plan individualisé;

- décider quels services seront fournis;

- refuser ou obtenir des services.

\section{Éléments d'un système de coordination des services de qualité}

Il existe des éléments clés qui font foi de services de qualité :

- un programme de coordination des services géré par un organisme qui ne fournit pas des services directement afin d'éviter les conflits d'intérêts dans la prise de décisions;

- un nombre raisonnable de clients;

- un système sans lacunes importantes en matière de services; 
- un partenariat regroupant tous les organismes fournissant des services aux personnes ayant un handicap et des liens avec des programmes de services plus généraux.

\section{Conditions nuisibles à l'efficacité d'une coordonnatrice de services}

Le nombre de personnes à desservir constitue le plus gros problème auquel doit faire face la coordonnatrice de services. Celle-ci peut avoir la charge de plus d'une centaine de personnes, parfois même jusqu'à trois cents.

Pour être efficace, la coordonnatrice de services a besoin d'avoir accès à toute une gamme de ressources communautaires. Dans certaines collectivités, les listes d'attente sont très longues. Les services doivent aussi être appropriés et enrichissants pour le client et sa famille et ils doivent être fondés sur des principes axés sur la valeur de la personne, comme une approche individualisée, le respect, la dignité, la protection contre le danger, l'intégration, l'autonomie et la productivité. Il peut y avoir des lacunes si les services disponibles ne remplissent pas ces conditions, souvent en raison d'un manque de ressources.

\section{Mesures prises par le gouvernement de l'Ontario pour assurer la prestation de services}

En avril 1997, le ministère des Services sociaux et communautaires a publié un document intitulé Pour des services au service des gens (MSSC 1997). Il s'agit d'un cadre de prestation des services communautaires et sociaux visant à mieux répondre aux besoins des enfants et des personnes ayant un handicap. Ce document établit des normes en vue d'aider les collectivités à collaborer et à 
utiliser les ressources disponibles d'une manière efficace. Dans un tel cadre, les personnes ayant un handicap et leur famille peuvent recevoir l'aide dont elles ont besoin sans se perdre dans le labyrinthe de services.Voici les points saillants de ce document:

- les services sont fournis aux personnes qui en ont le plus besoin;

- les services mettent l'accent sur l'intervention précoce et la prévention;

- l'accès aux services est simple et facile.

Ainsi, les services répondront davantage aux besoins des gens.

\section{Bibliographie}

MINISTÈRE DES SERVICES SOCIAUX ET COMMUNAUTAIRES (1997). Pour des services au service des gens, Communiqué de presse du 30 avril 1997.

\section{Note}

1. Le système de coordination des services géré par la YWCA de Sudbury a pour objectif de venir en aide aux personnes ayant un handicap de développement qui vivent dans les districts de Sudbury et Manitoulin.

Si vous désirez de plus amples informations sur le système de coordination des services, veuillez appeler le numéro de téléphone suivant (705) 673-4754 ou consulter le site WEB de la YWCA à l'adresse électronique suivante: www.cybersudbury.com et cliquez sur le lien «non profit». Pendant que vous y êtes, laissez-nous un courrier électronique à l'adresse suivante: sudywca@,vianet.on.ca. 